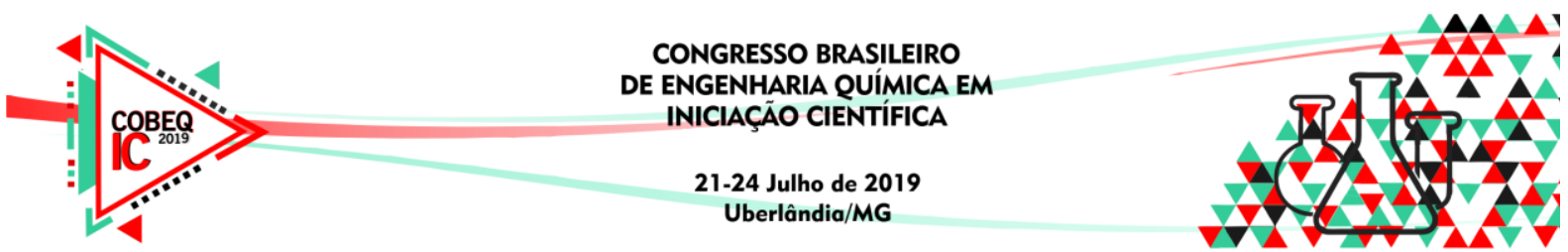

\title{
SÍNTESE DE NANOPARTÍCULAS DE FERRITAS E SUA APLICAÇÃO COMO ADSORVENTES NA REMOÇÃO DE AMOXICILINA EM ÁGUA
}

\author{
B. F. CAPISTRANO ${ }^{1}$, C. R. F. MARIANO ${ }^{1}$, R. F. N. MOURA ${ }^{1}$, T. N. S. OLIVEIRA ${ }^{1}$ e P. \\ M. A. CAETANO ${ }^{1,2}$ \\ ${ }^{1}$ Centro Universitário UNA \\ ${ }^{2}$ Centro de Desenvolvimento da Tecnologia Nuclear \\ E-mail para contato: patricia.caetano@una.prof.br
}

\begin{abstract}
RESUMO - A amoxicilina é um antibiótico amplamente usado na medicina humana e veterinária, em uma avaliação de risco de esgoto, esse fármaco foi identificado como um dos antibióticos mais comuns utilizados na medicina moderna que tem ecotoxicidade significativa. Consequentemente, é grande o interesse no desenvolvimento de novas tecnologias que sejam eficazes e possibilitem a remoção desse tipo de contaminante. No presente trabalho a remoção do antibiótico amoxicilina a partir de solução aquosa foi investigada a partir da adsorção, por ser um método simples e efetivo, utilizando-se nanoferritas de cobalto, zinco e cobre como materiais adsorventes. As ferritas foram sintetizadas pelo método de coprecipitação química e calcinadas a $400{ }^{\circ} \mathrm{C}$. Utilizou-se as técnicas de difração de raios $\mathrm{X}$ e adsorção/dessorção de nitrogênio pelo método BET na caracterização desses materiais. A eficiência de remoção da amoxicilina foi estudada por meio de medidas de espectroscopia na região UVVis, alcançando excelentes resultados quando comparado aos dados da literatura.
\end{abstract}

\section{INTRODUÇÃO}

A nanociência e a nanotecnologia podem ser aplicadas em praticamente todas as áreas da comunidade científica. Os nanomateriais possuem propriedades ópticas, mecânicas, térmicas, eletrônicas e químicas distintas das propriedades dos sólidos cristalinos típicos, devido a efeitos de tamanho e de superfície, tornando-se relevantes nas pesquisas de materiais que são utilizados como agentes antibacterianos (Kümmerer, 2009).

Em uma avaliação de risco de esgoto, a amoxicilina foi identificada como um dos antibióticos mais comuns utilizados na medicina moderna que tem ecotoxicidade significativa. Portanto, a remoção de amoxicilina em água antes da sua liberação para o ambiente é necessária (Kümmerer, 2009). A adsorção tem sido considerada um dos métodos mais eficazes para a remoção de antibióticos (Purceno et al., 2012). Entre os materiais adsorventes de nova geração desenvolvidos até agora, os nanoadsorventes magnéticos mostram-se os mais promissores (George et al., 2006). Nesse contexto, as nanopartículas de ferritas têm se destacado por apresentarem propriedades interessantes para serem aplicadas como adsorvente na remoção de amoxicilina em soluções aquosas (Ridley et al., 2015). As 


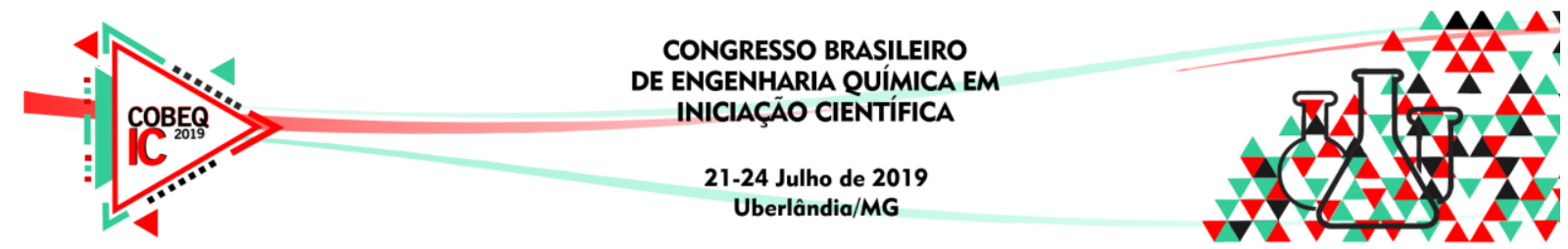

suas propriedades magnéticas permitem fácil separação de líquidos/sólidos e eventualmente a regeneração dos adsorventes.

Apesar das diversas pesquisas na área de remediação ambiental, há pouquíssimos trabalhos relacionando a capacidade de adsorção de ferritas de cobalto, zinco e cobre na remoção de amoxicilina. Desse modo, propôs-se neste projeto a síntese dessas nanoferritas e sua aplicação na adsorção de amoxicilina.

\section{METODOLOGIA}

As amostras foram sintetizadas pelo método de coprecipitação, que consiste em uma mistura de sais inorgânicos em ambiente aquoso e precipitação com hidrólise alcalina (Albuquerque et al., 2000). Utilizou-se nitratos de cobalto, cobre, zinco e ferro, e água destilada em quantidades estequiométricas e solução de hidróxido de sódio como agente precipitante. A solução dos nitratos permaneceu em agitação magnética por 1 hora, sendo posteriormente gotejada na solução de hidróxido de sódio, formando o precipitado. $\mathrm{O}$ precipitado foi lavado com água destilada até atingir o $\mathrm{pH}=7$, e por último com álcool etílico. $\mathrm{O}$ produto resultante foi secado à $80^{\circ} \mathrm{C}$ e calcinado em um forno mufla à $400{ }^{\circ} \mathrm{C}$.

A caracterização das amostras foi realizada por difração de raios X (DRX) visando determinar as fases presentes, conferir a estrutura cristalina e estimar o tamanho do cristalino. As medidas foram realizadas por um difratômetro do modelo Utimat, fabricação Rigaku, utilizando a radiação utilizando a radiação $\mathrm{K} \alpha$ do $\mathrm{Cu}$. A área superficial e o volume de poro das amostras foram estimados pela técnica de adsorção/dessorção de $\mathrm{N}_{2}$.

Os experimentos de adsorção foram conduzidos a temperatura ambiente, em $\mathrm{pH} 7$ utilizando-se $20 \mathrm{~mL}$ de solução de amoxicilina com concentração de $200 \mathrm{mgL}^{-1}$ e $20 \mathrm{mg}$ de ferrita. O material foi colocado em contato com a solução do fármaco por $24 \mathrm{~h}$, para garantir o equilíbrio. Em seguida, a mistura foi filtrada com um filtro de seringa $0,45 \mu \mathrm{m}$, e a solução resultante lida em um espectrofotômetro UV-Vis (T60 UV-Visible, PG Instruments). Para o monitoramento da remoção de amoxicilina da água, utilizou-se a absorção no comprimento de onda em $272 \mathrm{~nm}$. A obtenção da capacidade de adsorção (q), ou seja, a quantidade de amoxicilina adsorvida, em miligrama por grama de material, foi obtida a partir da Equação 1:

$$
q=\frac{\left(C_{i}-C_{f}\right) V}{m}
$$

onde $q$ é a quantidade de amoxicilina adsorvida $\left(\mathrm{mg}_{\mathrm{g}} \mathrm{g}^{-1}\right), C i$ e $C f$ a concentração inicial e final de amoxicilina $\left(\mathrm{mg} . \mathrm{L}^{-1}\right)$ respectivamente, $V$ o volume da fase aquosa (L) e $m$ a massa de adsorvente utilizada $(\mathrm{g})$.

\section{RESULTADOS E DISCUSSÕES}

\subsection{Difração de Raios - X}

Os padrões de difração de raios $\mathrm{X}$ das amostras de ferritas $\left(\mathrm{MFe}_{2} \mathrm{O}_{4}\right)$ sendo $\mathrm{M}=\mathrm{Co}, \mathrm{Cu}$ e Zn estão apresentados na Figura 1. 


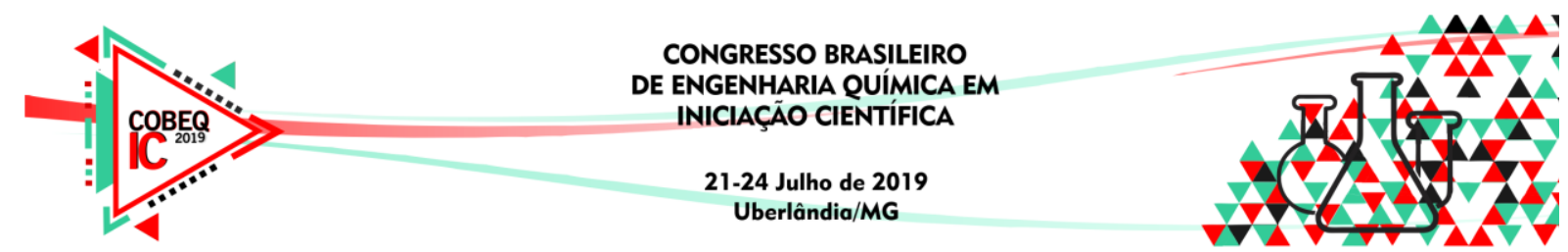

Figura 1 - Difratogramas das ferritas de $\mathrm{Zn}, \mathrm{Cu}$ e Co calcinadas a $400{ }^{\circ} \mathrm{C}$.

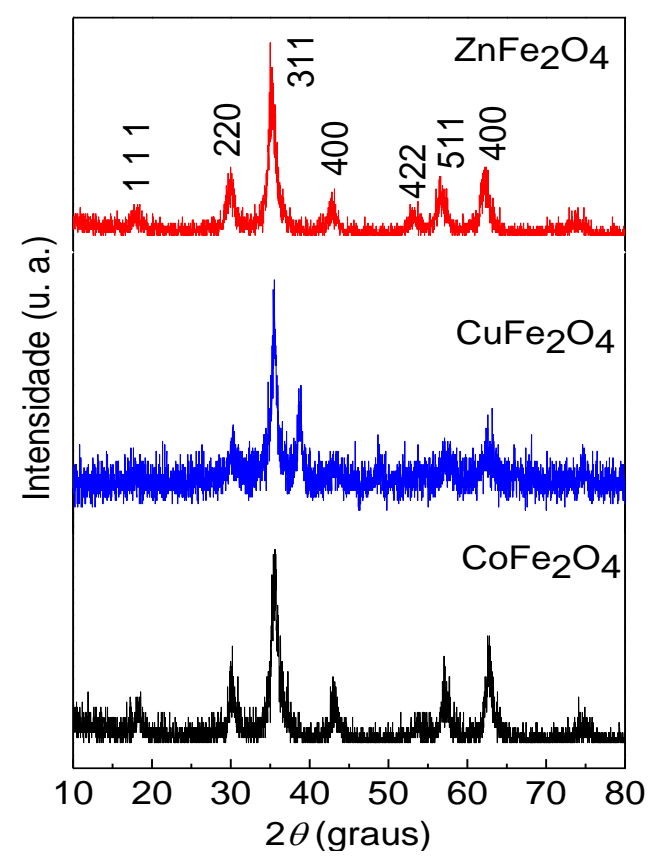

As medidas foram feitas usando passo de $2 \% \mathrm{~min}$., com varredura na faixa entre ângulos (20) de $10^{\circ}$ a $80^{\circ}$. Os difratogramas foram analisados por meio do programa Search Match para o tratamento dos dados e identificação de fases por comparação com os padrões de difração do banco de dados ICDD (International Centre for Diffraction Data). Todos os planos de reflexão observados nos difratogramas confirmam a formação da fase espinélio, característico das ferritas de $\mathrm{Co}, \mathrm{Cu}$ e $\mathrm{Zn}$. Observou- se picos de difração alargados devido ao tamanho reduzido dos materiais.O tamanho médio de partícula (D) foi estimado usando a equação de Scherrer (KLUG, 1956), equação 1:

$$
D=\frac{K \lambda}{\beta \cos \theta}
$$

onde D é o diâmetro médio $(\AA)$; K é o coeficiente de forma, que assume o valor de 0,9 devido a forma esférica da partícula; $\lambda$ é o comprimento de onda da radiação utilizada $(1,54056 \AA) ; \beta$ é a largura da meia altura do pico mais intenso do difratograma, que foi corrigido pelo padrão de quartzo; e $\theta$ é o ângulo de difração deste mesmo pico.

O diâmetro médio de partícula das amostras está apresentado na Tabela I. Este parâmetro tem uma grande importância, já que a adsorção é um fenômeno de superfície, e quanto menor o tamanho de partícula, maior a área superficial.

Tabela I - Diâmetro médio da nanopartícula

\begin{tabular}{c|c}
\hline Ferrita & Diâmetro médio (nm) \\
\hline $\mathrm{CoFe}_{2} \mathbf{O}_{4}$ & 8 \\
\hline $\mathrm{CuFe}_{2} \mathbf{O}_{4}$ & 12 \\
\hline $\mathrm{ZnFe}_{\mathbf{2}} \mathbf{O}_{4}$ & 9 \\
\hline
\end{tabular}




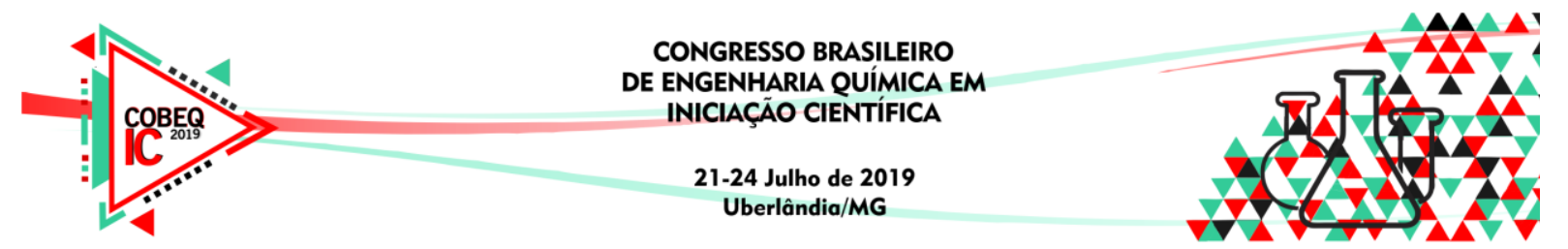

\subsection{Medida de área superficial por adsorção/dessorção de $\mathbf{N}_{2}$}

Os valores das áreas superficiais e do volume do poro foram determinados a partir da equação de BET e estão apresentados na Tabela II. É possível observar que a área superficial da ferrita de zinco é mais elevada, seguido pela ferrita de cobre e cobalto. Quanto maior a área superficial das nanopartículas, mais interessante se torna o material para adsorver o antibiótico, uma vez que terá uma área de contato maior com o mesmo.

Tabela II - Área superficial e volume médio do poro das nanoferritas

\begin{tabular}{c|c|c}
\hline Ferrita & Área superficial $\left(\mathbf{m}^{2} / \mathbf{g}\right)$ & Volume médio do poro $\left(\mathrm{cm}^{3} / \mathbf{g}\right)$ \\
\hline $\mathrm{CoFe}_{2} \mathbf{O}_{\mathbf{4}}$ & 73,41 & 0,22 \\
\hline $\mathrm{CuFe}_{2} \mathbf{O}_{\mathbf{4}}$ & 82,06 & 0,27 \\
\hline $\mathrm{ZnFe}_{2} \mathbf{O}_{\mathbf{4}}$ & 100,40 & 0,25 \\
\hline
\end{tabular}

\subsection{Estudo da Adsorção de Amoxicilina}

Foi realizada a varredura completa no espectrofotômetro UV-Vis de alíquotas de soluções padrão de amoxicilina com diferentes concentrações que está apresentado na Figura 2. Verificou-se que o pico mais intenso da amoxicilina foi em $272 \mathrm{~nm}$. Diante disso, executou-se a leitura das alíquotas nesse comprimento de onda e representou-se a relação das absorbâncias com as respectivas concentrações para a determinação da curva de calibração (Figura 3). Com objetivo de avaliar a capacidade adsortiva das amostras de ferritas, fez-se uma regressão linear para estabelecer equação da reta.

Figura 2 - Espectro de absorbância

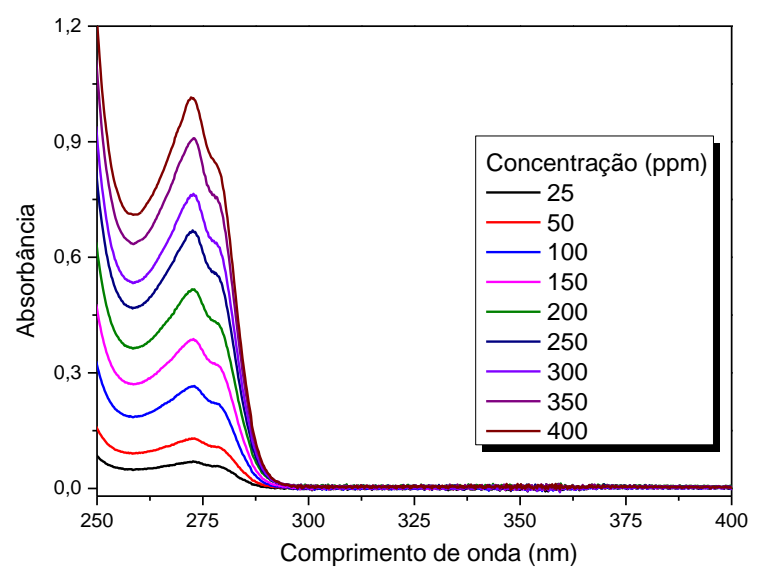

Figura 3 - Curva analítica da amoxicilina

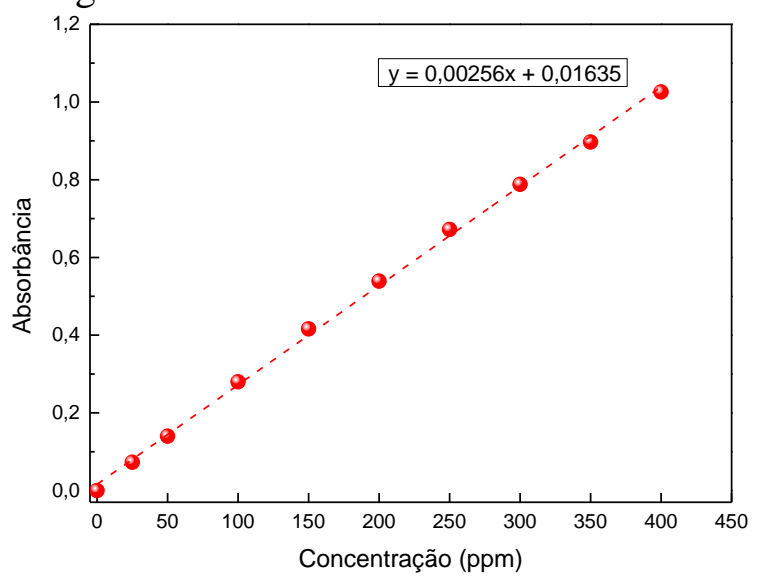

Os testes de adsorção foram realizados em duplicata, analisou-se as amostras no comprimento de onda de $272 \mathrm{~nm}$ e fez-se a comparação com a curva de absorbância da amoxicilina no mesmo comprimento de onda. Como pode ser observado na Figura 4 (a e b) houve um decréscimo no valor da absorbância das soluções de amoxicilina contendo ferritas de cobre e zinco, por conseguinte, conclui-se que ambas ferritas são promissoras na adsorção 


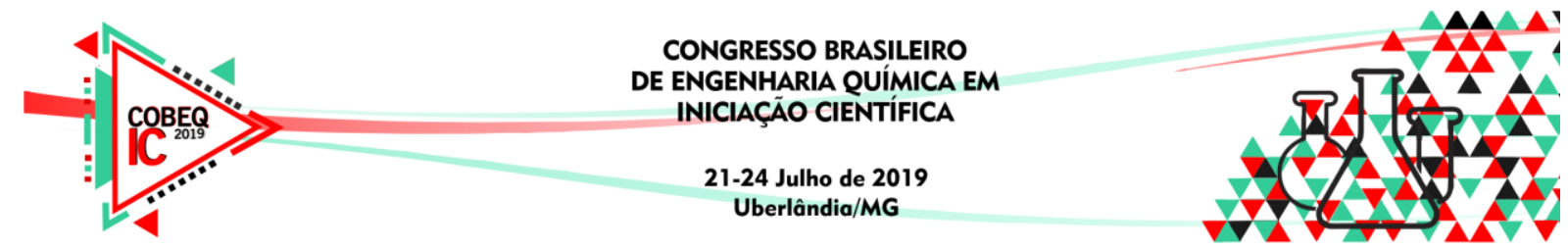

de amoxicilina em solução aquosa. Já as amostras contendo ferrita de cobre, foi observado uma elevação da absorbância, sugerindo uma possível degradação do fármaco (Figura 4c).

Figura 4 - Espectro de absorbância em função do comprimento de onda para as ferritas de $\mathrm{ZnFe}_{2} \mathrm{O}_{4}, \mathrm{CoFe}_{2} \mathrm{O}_{4}$ e $\mathrm{CuFe}_{2} \mathrm{O}_{4}$.
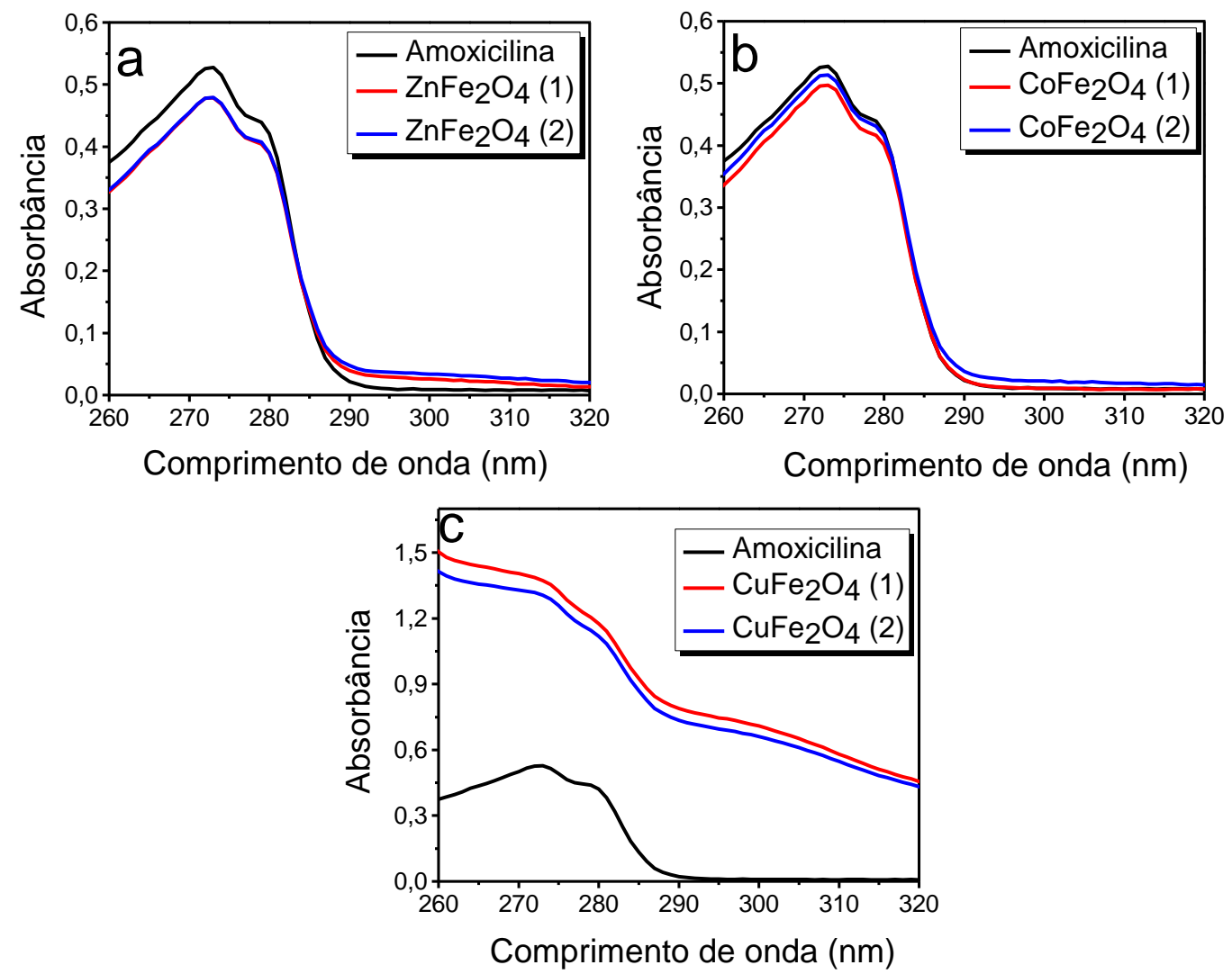

Após a determinação da concentração do fármaco utilizando-se a equação da reta proveniente da curva analítica (Figura 3), quantificou-se a quantidade de fármaco adsorvida de cada amostra, através da Equação 1. A ferrita de cobalto adsorveu $20 \mathrm{mg}_{\text {amoxicilina }} / \mathrm{g}_{\text {ferrita }}$ e a

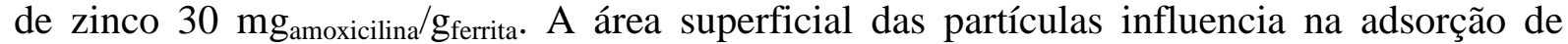
substâncias, logo, quanto maior a área, maior a capacidade de adsorção. Como esperado, analisando os resultados de BET a ferrita de zinco apresentou uma área superficial maior que a ferrita de cobalto.

Não há relatos da aplicação das ferritas de cobalto e zinco na adsorção de amoxicilina para comparação dos resultados, no entanto, na literatura, o uso de óxidos de ferro na adsorção foi relatado para tal aplicação. As ferritas apresentaram excelentes resultados na adsorção de amoxicilina quando comparadas com alumina, hematita e óxidos de ferro com proporções de 10, 20 e 50\% calcinados a 150, 200, 300 e $450{ }^{\circ} \mathrm{C}$ apresentado por (Pinto et al., 2016) e indicados na Figura 5. 


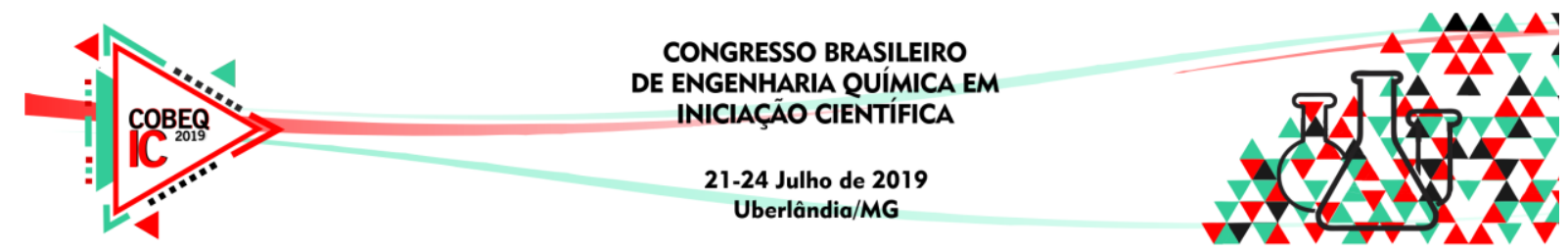

Figura 5 - Comparação da capacidade adsortiva das ferritas sintetizadas e compósitos de oxihidróxidos de ferro suportados em alumina.

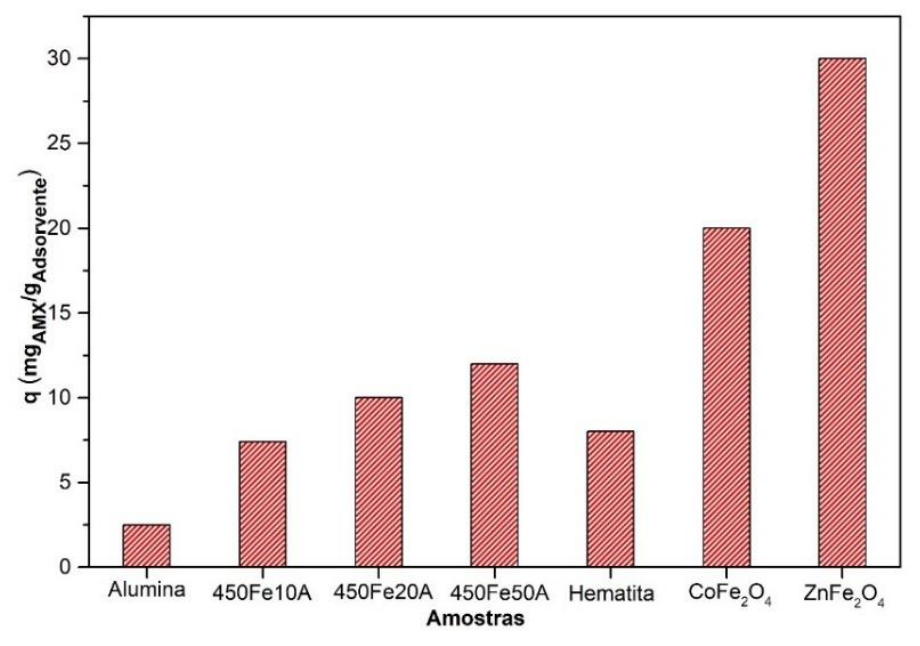

\section{CONCLUSÃO}

A análise detalhada dos parâmetros estruturais por difração de raios X, demonstraram que as nanopartículas de ferritas foram obtidas com êxito empregando-se o método de coprecipitação. As medidas de área superficial determinadas pelo método BET, mostraram que a área superficial variou de 73 a $100 \mathrm{~m}^{2} / \mathrm{g}$. Em relação ao estudo da adsorção avaliou-se que a ferrita de cobre não demonstrou capacidade adsortiva, mas possivelmente uma capacidade de degradação do fármaco. As ferritas de cobalto e zinco apresentaram boa capacidade adsortiva. A adsorção mais eficiente foi obtida para $\mathrm{ZnFe}_{2} \mathrm{O}_{4}$ com aproximadamente $9 \mathrm{~nm}$, removendo $30 \mathrm{mg}_{\text {amoxicilina }} / \mathrm{g}_{\text {ferrita. }}$. Este resultado mostra uma taxa de remoção significativamente maior que as descritas na literatura usando outros óxidos demonstrando o grande potencial das nanopartículas de ferrita de cobalto e zinco na remoção de antibióticos por adsorção.

\section{REFERÊNCIAS}

ALBUQUerQue, A. S. A.; ARDisson, J. D.; MACEDO, W. A. A.; ALVES, M. C. M. Nanosized powders of NiZn ferrite: synthesis, structure, and magnetism. Journal of Applied Physics, v. 87, n. 9, p. 4352, 2000.

GEORGE, M.; JOHN, A. M.; NAIR, S. S.; JOY, P. A.; ANANTHARAMAN, M.R. Finite size effects on the structural and magnetic properties of sol-gel synthesized NiFe2O4 powders. Journal of Magnetism Magnetic Materials, v. 302, p. 190-195, 2006.

PURCENO, A.D.; TEIXEIRA, A.P.C.; SOUZA, N.J.D.; FERNANDEZ-OUTON, L.E.; ARDISSON, J.D.; LAGO, R.M. Hybrid magnetic amphiphilic composites based on carbon nanotube/nanofibers and layered silicates fragments as efficient adsorbent for ethynilestradiol. Journal of Colloid and Interface Science, v. 379, p. 84-88, 2012.

RIDLEY, M. K.; MACHESKY, M. L.; KUBICKI, J. D. Experimental Study of Strontium Adsorption on Anatase Nanoparticles as a Function of Size with a Density Functional Theory and CD Model Interpretation. Langmuir, v. 31, p. 703- 713, 2015. 